\title{
Osteonecrosis of the jaws caused by bisphosphonate treatment and oxidative stress in mice
}

\author{
JOJI TAMAOKA $^{1 *}$, KAZUKI TAKAOKA ${ }^{1 *}$, HIROKAZU HATTORI $^{1}$, MIHO UETA $^{1}$, HANAKO MAEDA ${ }^{1}$, \\ MICHIYO YAMAMURA ${ }^{1}$, KOJI YAMANEGI ${ }^{2}$, KAZUMA NOGUCHI ${ }^{1}$ and HIROMITSU KISHIMOTO ${ }^{1}$ \\ Departments of ${ }^{1}$ Oral and Maxillofacial Surgery, and ${ }^{2}$ Pathology, Hyogo College of Medicine, \\ Nishinomiya, Hyogo 663-8501, Japan
}

Received February 3, 2018; Accepted September 6, 2018

DOI: $10.3892 /$ etm.2018.7076

\begin{abstract}
Aging is a significant risk factor for the development of bisphosphonate-related osteonecrosis of the jaws (BRONJ). Accumulating evidence suggests that bone aging is associated with oxidative stress (OS), and OS is associated with osteonecrosis. To elucidate the mechanisms of the onset of BRONJ, the present study focused on OS and the effects of treatment with the pro-oxidant DL-buthionine-(S,R)-sulfoximine (BSO), an oxidative stressor, on healing of a surgically induced penetrating injury of the palate. Six-week-old C57BL/6J mice were randomly divided into four groups ( $\mathrm{n}=5$ each) and treated with or without zoledronic acid (ZOL) and with or without BSO (experimental groups: ZOL, BSO, and ZOL+BSO; control group: saline solution). A penetrating injury of the midline palate was surgically created using a root elevator. ZOL $(250 \mu \mathrm{g} / \mathrm{kg} /$ day) was injected intraperitoneally every day from 7 days prior to the surgical treatment to 4 days following the surgical treatment. BSO $(500 \mu \mathrm{g} / \mathrm{kg} /$ day $)$ was administered 7 days prior to the surgical treatment as a single intraperitoneal injection. The maxillae were harvested at 5 days following the surgical treatment for histological and histochemical studies. The presence of empty osteocyte lacunae in the palatal bone was increased by ZOL and BSO treatment. The highest number of empty osteocyte lacunae was observed in the ZOL+BSO group. The number of tartrate-resistant acid phosphatase-positive cells was decreased by ZOL treatment and increased by BSO treatment. The number of canaliculi per osteocyte lacuna was significantly decreased by BSO treatment. The mineral apposition rate was significantly lower in the treatment groups than the control group. Bisphosphonates and OS suppressed bone turnover. The present study has demonstrated that BSO
\end{abstract}

Correspondence to: Dr Kazuki Takaoka, Department of Oral and Maxillofacial Surgery, Hyogo College of Medicine, 1-1 Mukogawa-cho, Nishinomiya, Hyogo 663-8501, Japan

E-mail:ktaka@hyo-med.ac.jp

*Contributed equally

Key words: osteonecrosis, bisphosphonate, oxidative stress treatment affects osteocytes, and OS in osteocytes exacerbates impairment of the osteocytic canalicular networks. As a result, bisphosphonates and OS may induce osteonecrosis following invasive dentoalveolar surgery. OS has been identified as an additional risk factor for the development of BRONJ.

\section{Introduction}

Bisphosphonates (BPs) accelerate osteoclast apoptosis. The mechanism comprises strong inhibition of bone resorption $(1,2)$. BPs are effective for the treatment of osteoporosis, Paget's disease, multiple myeloma, hypercalcemia of malignancy and bone metastases from breast cancer and prostate cancer (3-5). However, BP-related osteonecrosis of the jaws (BRONJ) is a serious problem in patients treated with BPs $(6,7)$. The risk factors for BRONJ comprise drug-associated, local and demographic/systemic factors. Drug-associated risk factors include the potency of the specific BP. Zoledronic acid (ZOL) is the most potent BP (8). It also carries the highest incidence of BRONJ (9). The drugs that have primarily been used in evaluated studies $(10,11)$ on BRONJ arising in rodents under BP therapy following tooth extraction were ZOL and alendronate (12). Local risk factors include dentoalveolar surgery, tooth extraction, periapical surgery and periodontal surgery including osseous injury. Systemic risk factors include corticosteroid therapy, diabetes and chemotherapeutic drugs (13). A number of animal models of BRONJ associated with risk factors such as corticosteroid therapy (14), vitamin D deficiency (15) and diabetes (16) combined with tooth extraction have also been established.

Aging is an additional significant risk factor for the development of BRONJ (17-19). Bone aging is associated with oxidative stress (OS) as demonstrated in both human studies and animal models (20-23). OS occurs as result of overproduction of reactive oxygen species (ROS) that is not balanced by an adequate level of antioxidants (24). BP treatment can produce OS (25), and continued local inflammation, either with or without an associated infective process (26-29), can produce ROS. Khandelwal et al (30) previously reported that treatment with ZOL increased OS in the human breast cancer cell line MCF-7, and this increase in OS was reversed by antioxidants. The authors reported that ZOL can induce a dose-dependent but irreversible autophagy by its effect on the mevalonate 
pathway and OS (30). It has also been demonstrated that OS is associated with osteonecrosis (31-33). Ichiseki et al (34) have demonstrated that a single intraperitoneal injection of the pro-oxidant DL-buthionine-(S,R)-sulfoximine (BSO) $(500 \mathrm{mg} / \mathrm{kg})$, an oxidative stressor, in rats was by itself sufficient to induce osteonecrosis. Osteonecrosis in the femoral head was confirmed at 5 (2 of 20 rats, 10\%), 7 (7 of 20 rats, 35\%), and 14 days following BSO injection (8 of 20 rats, 40\%) (34). To the best of our knowledge, no reports to date have described animal models of BRONJ associated with OS. To elucidate the mechanisms of the onset of BRONJ, the present study focused on OS and investigated the effects of ZOL and BSO in a short term on healing of surgically created palatal defects.

\section{Materials and methods}

Animal handling. The present study was approved by the Ethics Committee of the Hyogo College of Medicine (Hyogo, Japan; approval number 16-078). Male 5-week-old C57BL/6J mice ( $n=40$; body weight, 18-21 g) were obtained from Japan SLC, Inc. (Hamamatsu, Japan). The animals were housed in a temperature-, humidity-, and light-controlled room $\left(23 \pm 3^{\circ} \mathrm{C}\right.$; $55 \pm 15 \%$; 12-h light-dark cycle). Food and water were available ad libitum.

Agents. ZOL [2-(imidazol-1-yl)-1-hydroxyethylidene-1,1-BP] and BSO (DL-buthionine-(S,R)-sulfoximine) were purchased from Sigma-Aldrich; Merck KGaA (Darmstadt, Germany).

Experimental methods and design. Following 1 week of acclimatization, the 6-week-old mice were randomly divided into four groups ( $\mathrm{n}=5$ each) and treated with or without ZOL and with or without BSO (experimental groups: ZOL, BSO, and ZOL+BSO; control group: saline solution; Fig. 1A). A penetrating injury of the midline palate was surgically created using a root elevator under anesthesia with $2 \%$ isoflurane (Pfizer Japan, Inc., Tokyo, Japan) (Fig. 1B). Dentoalveolar surgery is a risk factor for the development of BRONJ in patients receiving BPs (6). Therefore, tooth extraction is commonly used to induce osteonecrosis in animal models. In the present study, a penetrating injury of the midline palate was surgically created using a root elevator as a less invasive surgery than tooth extraction to minimize the suffering or distress of eating with missing teeth. No problems were associated with the presence of root fragments in the extraction socket. ZOL $(250 \mu \mathrm{g} / \mathrm{kg} /$ day $)$ and saline solution at the same dosage volume were injected intraperitoneally from 7 days prior to the surgical treatment to 4 days following the surgical treatment. The dosage and duration of administration of ZOL was based on the protocols described previously by Kobayashi et al (35). BSO (500 $\mu \mathrm{g} / \mathrm{kg} /$ day) was administered 7 days prior to the surgical treatment as a single intraperitoneal injection. The total maxillae were then harvested en bloc 5 days following the surgical treatment (Fig. 1C).

Bone histomorphometric analysis. To determine the bone histomorphometric parameters of mouse femurs, the femurs from 4 mice in each group were harvested at the same time as the total maxillae, stored in $70 \%$ ethanol at $4^{\circ} \mathrm{C}$, and analyzed using a micro-CT scanner (Scan Xmate-L090;
Comscan Techno Co., Ltd., Kanagawa, Japan). Scanning was conducted at $75 \mathrm{kV}$ and $105 \mathrm{~mA}$ with a spatial resolution of $\sim 9.073 \mathrm{~mm} / \mathrm{pixel}$. For quantitative analysis, the bone volume (BV/TV), trabecular thickness (Tb.Th), trabecular number (Tb.N), and trabecular separation (Tb.Sp) were determined using TRI/3D-BON software version R9 (RATOC System Engineering Co., Ltd., Tokyo, Japan).

Measurement of serum $8-O H d G$. Blood samples $(0.8 \mathrm{ml})$ were collected from the left ventricle under anesthesia with $2 \%$ isoflurane for serum analysis $6 \mathrm{~h}$ following treatment with or without ZOL and with or without BSO $(n=5)$, and then they were euthanized by cervical dislocation. The 8-hydroxy-2'-deoxyguanosine (8-OHdG) level has been widely analyzed as a marker of an individual's OS (36). The serum concentration of $8-\mathrm{OHdG}$ was measured using a highly sensitive ELISA kit (Highly Sensitive 8-OHdG Check ELISA kit; cat. no. KOG-HS10/E; Japan Institute for the Control of Aging; Nikken SEIL Co., Ltd., Shizuoka, Japan) according to the manufacturer's protocol. The absorbance at $405 \mathrm{~nm}$ was determined using a microplate reader (Benchmark Plus ${ }^{\mathrm{TM}}$ Microplate Spectrophotometer; Bio-Rad Laboratories, Inc., Hercules, CA, USA).

Histopathology. The mice were euthanized by cervical dislocation under anesthesia, induced by the inhalation of $5 \%$ isoflurane. The maxillae were harvested from the control, $\mathrm{ZOL}, \mathrm{BSO}$ and $\mathrm{ZOL}+\mathrm{BSO}$ groups. The tissue specimens were immediately placed in $10 \%$ neutral buffered formalin at room temperature for $24 \mathrm{~h}$ and decalcified in $10 \%$ ethylenediaminetetraacetic acid at room temperature for 2 weeks. Paraffin sections (4- $\mu$ m-thick) were cut using conventional methods and stained with hematoxylin and eosin (H\&E). Sections were stained with hematoxylin for $5 \mathrm{~min}$, washed with distilled water, dipped in $0.1 \%$ ammonium solution several times and washed again with $100 \%$ alcohol. The samples were stained with $1 \%$ eosin solution for $20 \mathrm{sec}$ at room temperature. The region of interest (ROI) corresponded to the palatal bone including the surgically perforated part and alveolar bone. The total numbers of osteocyte lacunae and empty osteocytic lacunae were counted in four non-overlapping defined ROIs at a magnification of x 200 under light microscopy. Paraffin sections were cut again and stained with Alcian blue for $30 \mathrm{~min}$ at room temperature to identify cartilage and bone under light microscopy (magnification, x200).

Tartrate-resistant acid phosphatase (TRAP) staining was performed as described previously (16). Briefly, samples were placed in $0.2 \mathrm{M}$ acetate buffer [0.2 $\mathrm{M}$ sodium acetate and $50 \mathrm{mM} \mathrm{L(+)} \mathrm{tartaric} \mathrm{acid} \mathrm{in} \mathrm{double-distilled} \mathrm{water;}$ $\mathrm{pH} 5.0]$ for $20 \mathrm{~min}$ at room temperature. The sections were then incubated with $0.5 \mathrm{mg} / \mathrm{ml}$ naphthol AS-MX phosphate (Sigma-Aldrich; Merck KGaA) and $1.1 \mathrm{mg} / \mathrm{ml}$ Fast Red TR Salt (Sigma-Aldrich; Merck KGaA) in $0.2 \mathrm{M}$ acetate buffer for $1-4 \mathrm{~h}$ at $37^{\circ} \mathrm{C}$ until the osteoclasts appeared bright red (37). The number of multinuclear TRAP-positive cells was counted in four non-overlapping defined ROIs at a magnification of x200 under light microscopy.

For the canaliculi structure analysis, the bone sections were incubated at room temperature for $30 \mathrm{~min}$ in silver staining solution in the dark. The silver staining solution was prepared 

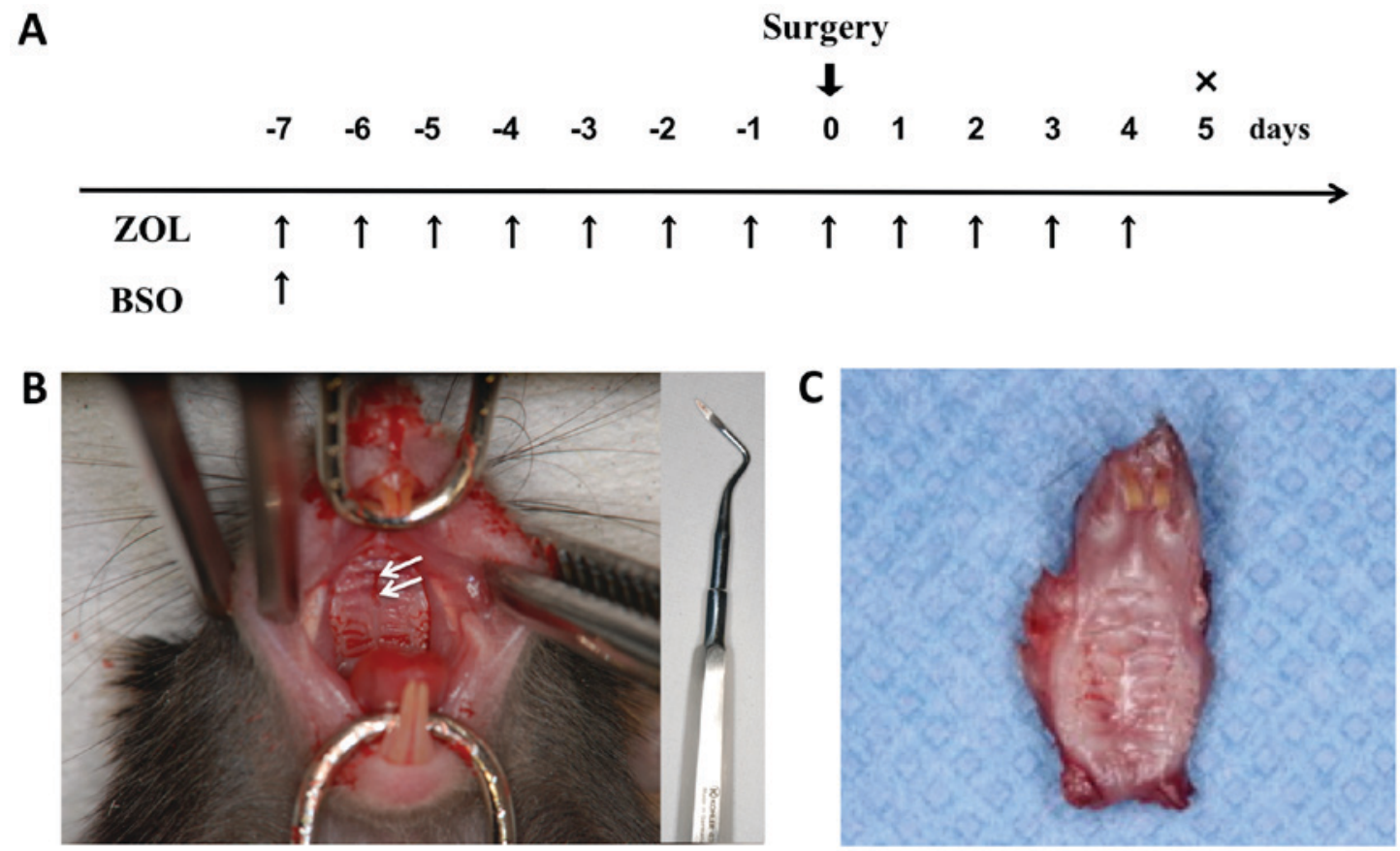

Figure 1. Treatment schedule. ZOL $(250 \mu \mathrm{g} / \mathrm{kg} / \mathrm{day})$ and saline solution at the same dosage volume were injected intraperitoneally from 7 days prior to the surgically created defect to 4 days following the surgical treatment. (A) BSO was administered 7 days prior to the surgical treatment as a single intraperitoneal injection (arrows indicate injections, $\mathrm{x}$ indicates euthanasia). (B) A defect of the midline palate was surgically created using a root elevator as the surgical treatment (arrows). (C) The total maxillae were harvested en bloc and examined macroscopically and microscopically. ZOL, zoledronic acid; BSO, DL-buthionine-(S,R)-sulfoximine.

by combining silver nitrate ( 2 volumes of $50 \%$ aqueous solution; Wako Pure Chemical Industries, Ltd., Osaka, Japan) and formic acid (1 volume of $1 \%$ solution containing $2 \%$ gelatin). The sections were washed in distilled water and transferred to a 5\% aqueous sodium thiosulfate solution at room temperature for $5 \mathrm{~min}$. The number of canaliculi per osteocyte lacuna (N.Ot.Ca/Ot.Lc.) were counted in 10 cells in 4 randomly selected non-overlapping defined ROIs at x400 magnification under light microscopy.

Dynamic calcein labeling. At 9 and 2 days prior to euthanasia, 5 mice in each group were administered an intraperitoneal injection of $10 \mathrm{mg} / \mathrm{kg}$ calcein (Dojindo Molecular Technologies, Inc., Kumamoto, Japan) for double labeling. Non-fixed frozen sections (6- $\mu \mathrm{m}$ thickness) from the maxilla were prepared with an adhesive film and a disposable tungsten carbide blade [Cryofilm type 2C(9) and SL-T30 (UF), respectively; SECTION LAB Co., Ltd., Hiroshima, Japan] according to the method described by Kawamoto T and Kawamoto K (38), and calcein labeling was assessed. An Olympus fluorescent microscope (Olympus Corporation, Tokyo, Japan) was used, and the calcein double labels were analyzed with an excitation wavelength of $485 \mathrm{~nm}$ and an emission wavelength of $510 \mathrm{~nm}$ at a magnification of $\mathrm{x} 200$. The mineral apposition rate (MAR; $\mu \mathrm{m} /$ day), defined as the distance between the midpoints of the double label divided by the number of days between calcein injections, was also measured (39).

Statistical analysis. All data are expressed as mean + or \pm standard deviation. Statistical analysis was performed using one-way analysis of variance followed by Bonferroni's multiple comparison test (SPSS version 22.0 software; IBM
Corp., Armonk, NY, USA). $\mathrm{P}<0.05$ was considered to indicate a statistically significant difference.

\section{Results}

Body weight. There were no significant differences in body weight among the control, ZOL, BSO and ZOL+BSO groups during the experimental period (data not shown).

Macroscopic evaluation. In all groups, the surgical perforation exhibited complete mucosal closure by the end of the study. No open wound or bone exposure was noted in any group.

Bone histomorphometric analysis of the distal femur. Bone histomorphometric analysis was used to determine BV/TV, Tb.Th, Tb.N, and Tb.Sp (Table I). In the inter-group comparison, significant differences in Tb.N (ZOL group, 4.45 $\pm 0.651 / \mathrm{mm}$; BSO group, 3.07 $\pm 0.421 / \mathrm{mm}$; Table I) and Tb.Sp (ZOL group, $197.40 \pm 37.41 \mu \mathrm{m}$; BSO group, $298.43 \pm 41.63 \mu \mathrm{m}$; Table I) were observed between the ZOL and BSO groups.

Measurement of serum 8-OHdG. The 8-OHdG level was significantly increased by BSO treatment (control group, $0.171 \pm 0.037 \mathrm{ng} / \mathrm{ml}$; BSO group, $0.213 \pm 0.033 \mathrm{ng} / \mathrm{ml}$; Table II).

Histological evaluation. Sections of maxilla including the surgically perforated part were stained with H\&E and examined histologically in all four groups. Complete epithelial coverage was noted in all groups. Wound healing in the palate was assessed to investigate the effect of ZOL and BSO on bone healing (Fig. 2A). The cartilage was less completely formed around the surgically perforated part in the treatment 
Table I. Bone histomorphometric analysis of the distal femur.

\begin{tabular}{lrccc}
\hline & \multicolumn{3}{c}{ Parameter } \\
\cline { 2 - 4 } Group & BV/TV $(\%)$ & Tb.Th $(\mu \mathrm{m})$ & Tb.N $(1 / \mathrm{mm})$ & Tb.Sp $(\mu \mathrm{m})$ \\
\hline Control & $11.33 \pm 2.56$ & $31.21 \pm 3.36$ & $3.61 \pm 0.53$ & $250.30 \pm 46.68$ \\
ZOL & $14.05 \pm 2.48$ & $31.52 \pm 1.85$ & $4.45 \pm 0.65^{\mathrm{a}}$ & $197.40 \pm 37.41^{\mathrm{a}}$ \\
BSO & $9.60 \pm 1.23$ & $31.26 \pm 1.37$ & $3.07 \pm 0.42$ & $298.43 \pm 41.63$ \\
ZOL+BSO & $12.10 \pm 2.23$ & $31.13 \pm 1.64$ & $3.88 \pm 0.56$ & $230.47 \pm 39.76$ \\
\hline
\end{tabular}

Data are expressed as mean \pm standard deviation. ${ }^{\mathrm{a}} \mathrm{P}<0.05$ vs. BSO. BV/TV, bone volume; Tb.Th, trabecular thickness; Tb.N, trabecular number; Tb.Sp; ZOL, zoledronic acid; BSO, DL-buthionine-(S,R)-sulfoximine.

Table II. Changes in serum 8-OHdG concentration.

\begin{tabular}{lc}
\hline Group & $8-\mathrm{OHdG}(\mathrm{ng} / \mathrm{ml})$ \\
\hline Control & $0.171 \pm 0.037$ \\
ZOL & $0.169 \pm 0.028$ \\
BSO & $0.213 \pm 0.033^{\mathrm{a}}$ \\
ZOL+BSO & $0.201 \pm 0.036$ \\
\hline
\end{tabular}

Data are expressed as mean \pm standard deviation. ${ }^{\mathrm{a}} \mathrm{P}<0.05$ vs. control. 8-OHdG, 8-hydroxy-2'-deoxyguanosine; ZOL, zoledronic acid; BSO, DL-buthionine-(S,R)-sulfoximine.

groups, especially in the BSO group, than in the control group (Fig. 2B and C). Fibrous connective tissue was present around the surgical perforation in the treatment groups, especially in the ZOL+BSO group (Fig. 2B). Areas of necrotic bone with empty osteocyte lacunae were observed in the palatal bone around the surgical perforation in the ZOL+BSO group (Fig. 2D).

The ratio of the number of empty osteocytic lacunae to the total number of osteocyte lacunae was used to calculate the percentage of dead osteocytes in the alveolar bone and palatal bone. The number of empty osteocyte lacunae in the alveolar bone (non-surgery area) and palatal bone (surgery area) was evaluated (Fig. 2E and F). The number of empty osteocyte lacunae in the alveolar bone was significantly increased by treatment with ZOL+BSO (proportion of empty osteocyte lacunae among total osteocyte lacunae: control group, $18.0 \pm 1.1 \%$; ZOL group, $36.1 \pm 6.3 \%$; BSO group, $29.4 \pm 7.9 \%$; $\mathrm{ZOL}+\mathrm{BSO}$ group, $35.9 \pm 9.8 \%$ ). The number of empty osteocyte lacunae in the palatal bone was significantly increased by treatment with ZOL+BSO (proportion of empty osteocyte lacunae among total osteocyte lacunae: control group, $24.8 \pm 9.7 \%$; ZOL group, $43.5 \pm 15.6 \%$; BSO group, $39.7 \pm 11.1 \%$; ZOL+BSO group, $53.9 \pm 20.5 \%$ ). More empty osteocyte lacunae were present in the palatal bone around the surgical perforation than in the alveolar bone in all groups.

Osteoclast activity. TRAP-positive osteoclasts were present on the bone surface of the palatal bone (Fig. 3A). The number of TRAP-positive osteoclasts was decreased by ZOL treatment and increased by BSO treatment (number of TRAP-positive cells: Control group, 5.5 \pm 1.4 ; ZOL group, 3.0 \pm 2.1 ; BSO group, $6.6 \pm 2.4 ; \mathrm{ZOL}+\mathrm{BSO}$ group, $6.0 \pm 1.2)$. There were no significant differences among the groups (Fig. 3B).

Osteocytic canalicular morphology. AgNOR staining was performed to investigate morphological changes in the palatal bone (Fig. 4A). The N.Ot.Ca/Ot.Lc. was significantly decreased by $\mathrm{BSO}$ treatment and $\mathrm{ZOL}+\mathrm{BSO}$ treatment compared with the control. N.Ot.Ca/Ot.Lc. was also markedly decreased by ZOL treatment (Fig. 4B).

Bone dynamic parameters. Following calcein administration, the double calcein-green labels were observed in the bones of the mice. Two clear calcein-labeled lines were recognizable in the newly formed bone around the palatal bone (Fig. 5A). The MAR was significantly lower in the treatment groups than in the control group (Fig. 5B).

\section{Discussion}

In the present study, ZOL treatment tended to increase the BV/TV and Tb.N of the femur and decrease the Tb.Sp in mice. These findings may suggest that the experimental protocol generated the expected anticatabolic effect of ZOL treatment in bone. The bone remodeling rate is thought to be higher in the jaw than femur throughout life (40). Therefore, suppression of bone turnover by ZOL treatment may have more profound effects on the jaw bones than long bones. The BSO group exhibited significantly increased levels of $8-\mathrm{OHdG}$ as a marker of an individual's OS compared with the control group.

Bone tissue is continuously renewed by bone remodeling, which comprises a dynamic interplay among bone cells including osteoclasts, osteoblasts, and osteocytes (41). BPs induce osteoclast apoptosis, which can be recognized by morphological changes in osteoclasts both in vitro (42-44) and in vivo (42). The number of multinuclear TRAP-positive cells was evaluated as osteoclasts. The number of TRAP-positive cells was lower in the ZOL group than in the control group. This result suggests that BP treatment may serve important roles in the inhibition of bone turnover by osteoclasts in the bone surgery area. Otherwise, only BSO treatment increased the number of TRAP-positive osteoclasts. There was a difference in the number of TRAP-positive osteoclasts between 
A

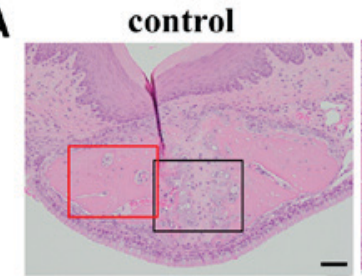

B

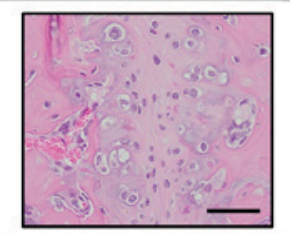

C

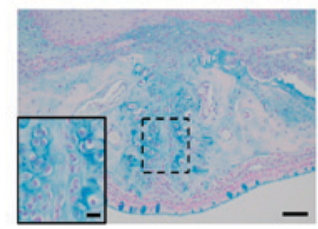

D

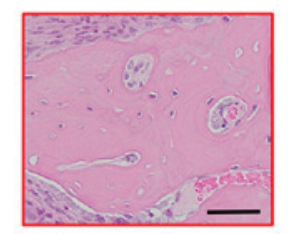

ZOL
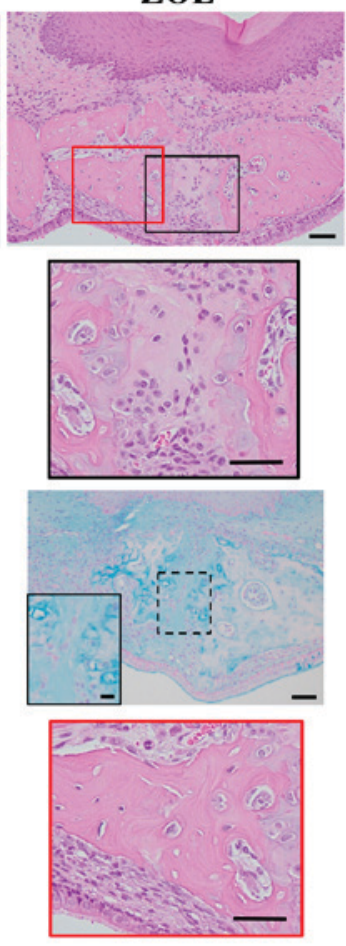
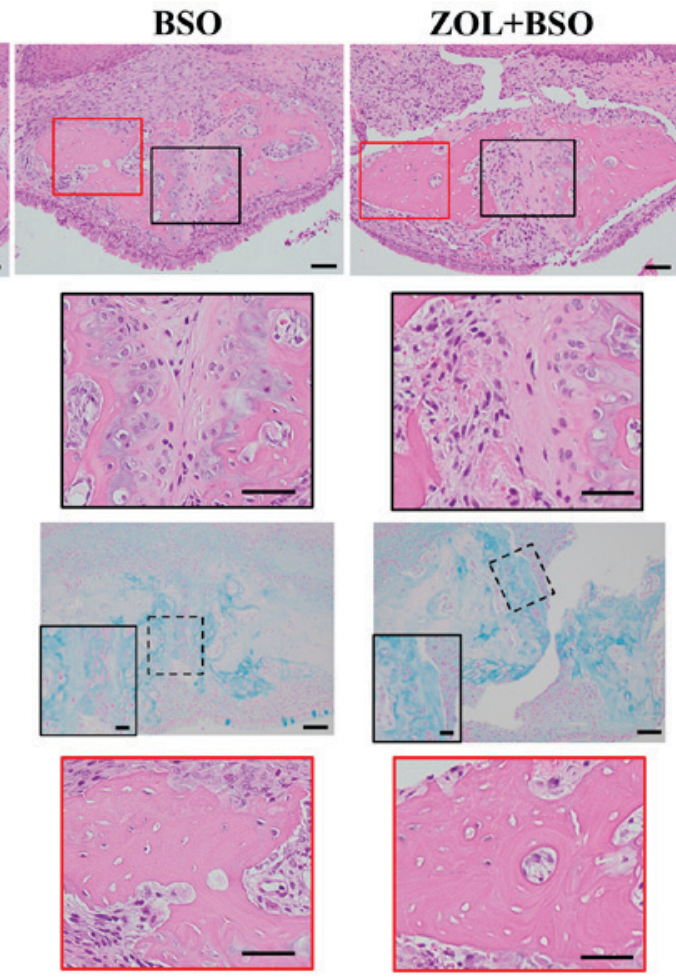

$\mathbf{E}$

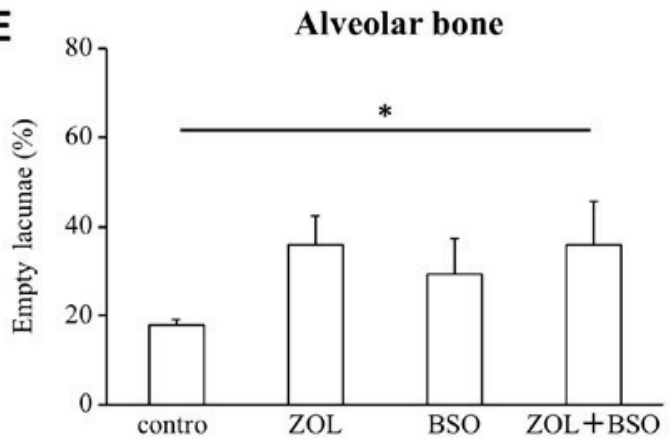

$\mathbf{F}$

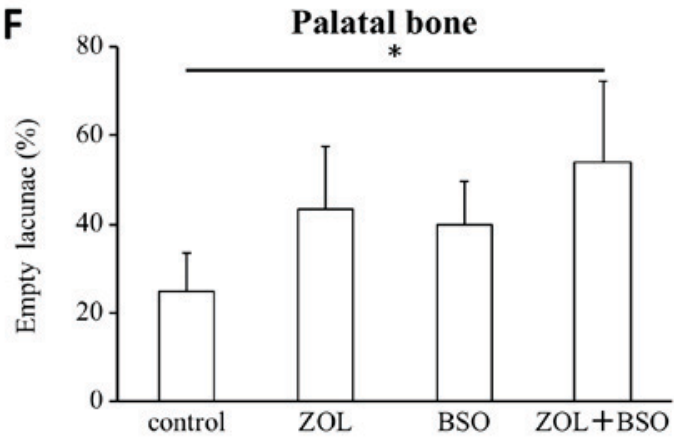

Figure 2. Analysis of palatal bone including the surgical perforation and alveolar bone. (A) Photomicrographs of the surgical perforation. Hematoxylin and eosin stain; original magnification, $\mathrm{x} 200$. Scale bar, $50 \mu \mathrm{m}$. (B) Photomicrographs of magnified black square area in (A). Scale bar, $50 \mu \mathrm{m}$. (C) Photomicrographs of the surgical perforation. Alcian blue staining; original magnification, $\mathrm{x} 200$. Scale bar, $50 \mu \mathrm{m}$. Insets present the higher magnification of the dotted square area. Scale bar, $10 \mu \mathrm{m}$. (D) Photomicrographs of magnified red square area in (A). Scale bar, $50 \mu \mathrm{m}$. The ratio of the number of empty osteocytic lacunae to the total number of osteocyte lacunae was used to calculate the percentage of dead osteocytes in the (E) alveolar and (F) palatal bone. The numbers of total osteocyte lacunae and empty osteocytic lacunae were counted in four non-overlapping defined regions of interest at a magnification of $\mathrm{x} 200$. $^{*} \mathrm{P}<0.05$. ZOL, zoledronic acid; BSO, DL-buthionine-(S,R)-sulfoximine.

the BSO and ZOL groups. These results suggest that BSO treatment is unrelated to osteoclast apoptosis. OS occurs as a result of ROS overproduction. ROS have opposite effects on osteoclast and osteoblast activity. ROS induce the apoptosis of osteoblasts and osteocytes and activate the differentiation of osteoclasts (24).

It was also observed that there were significantly more empty osteocyte lacunae in the alveolar bone and palatal bone in the ZOL+BSO group than in the control group. Empty osteocyte lacunae were prone to increase by treatment with ZOL. This implies that ZOL may have the potential to exacerbate bone damage. In all groups, the number of empty osteocyte lacunae was higher around the surgically created defect in the palatal than alveolar bone. When bone becomes necrotic, bone repair is initiated by osteoclasts. However, necrotic bone persists in the region because of osteoclast suppression by ZOL. This is why dentoalveolar surgery is a risk factor for the development of BRONJ in patients receiving BPs (16). Empty osteocyte lacunae in the alveolar bone and palatal bone were increased by single treatment with ZOL or BSO, but not significantly. Empty osteocyte lacunae were significantly increased by combined treatment with ZOL and BSO. These results indicate that single treatment with $\mathrm{ZOL}$ or BSO did not induce ONJ in the present study. This may have been caused by shortages in the dosage and duration of administration of these drugs. Long-term BP treatment seems to be an important risk factor for BRONJ $(45,46)$. However, combined treatment with ZOL and BSO induced ONJ. The present results suggest that both of these agents contribute to the onset of BRONJ in a short term. Future experiments will aim to confirm the results of the current study by determining whether inhibition of OS may prevent $\mathrm{ZOL}+\mathrm{BSO}$-induced ONJ.

Osteocytes are embedded in the bone matrix within a network of lacunae and canaliculi. Osteocytes use their 
A

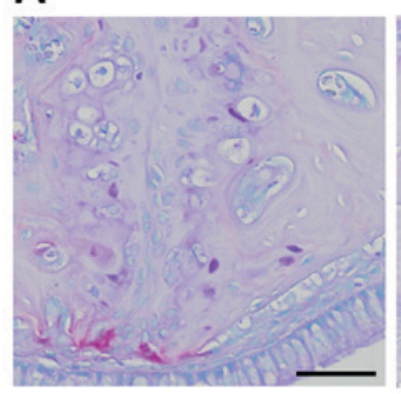

ZOL

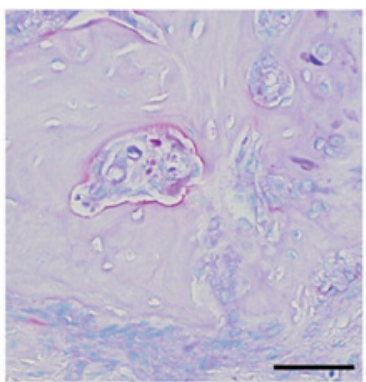

BSO

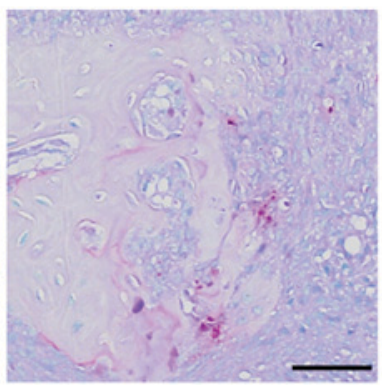

ZOL+BSO

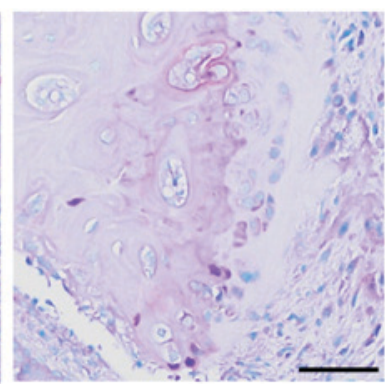

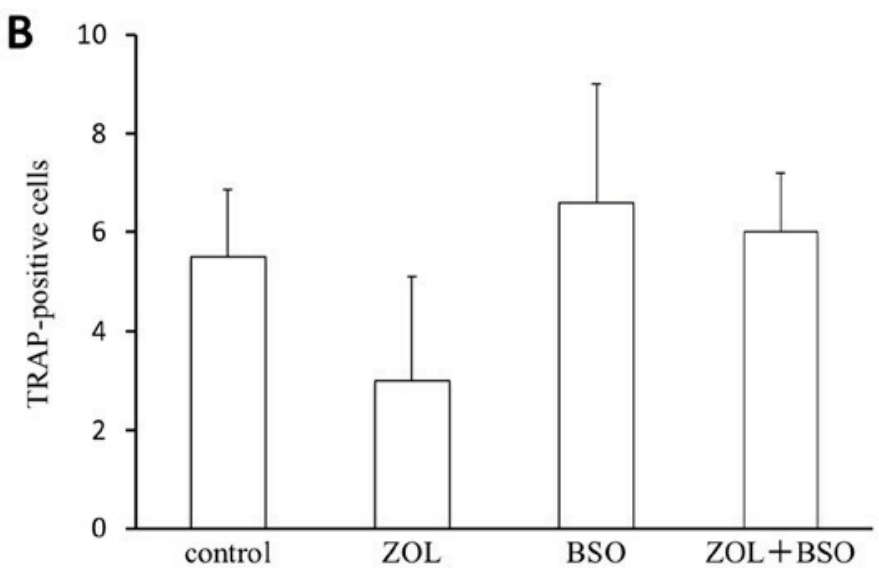

Figure 3. TRAP-stained sections. (A) TRAP-positive cells on the bone surface in the palatal bone. Original magnification, $\mathrm{x} 200$. Scale bar, $50 \mu \mathrm{m}$. (B) The number of multinuclear TRAP-positive cells was counted in the palatal bone at a magnification of x200. TRAP, tartrate-resistant acid phosphatase; ZOL, zoledronic acid; BSO, DL-buthionine-(S,R)-sulfoximine.

A control

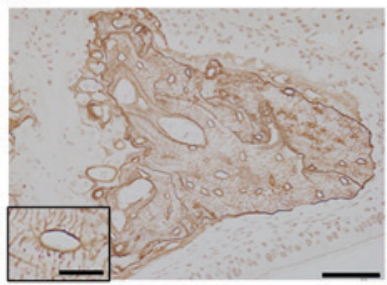

ZOL

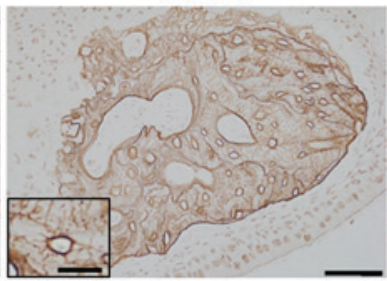

BSO

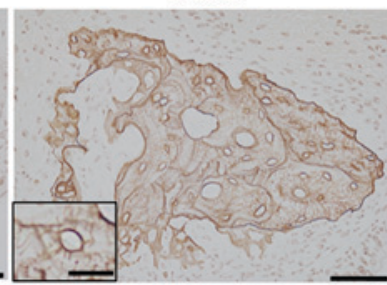

ZOL+BSO

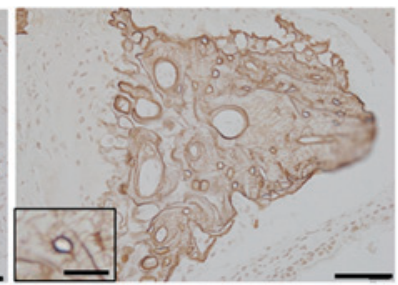

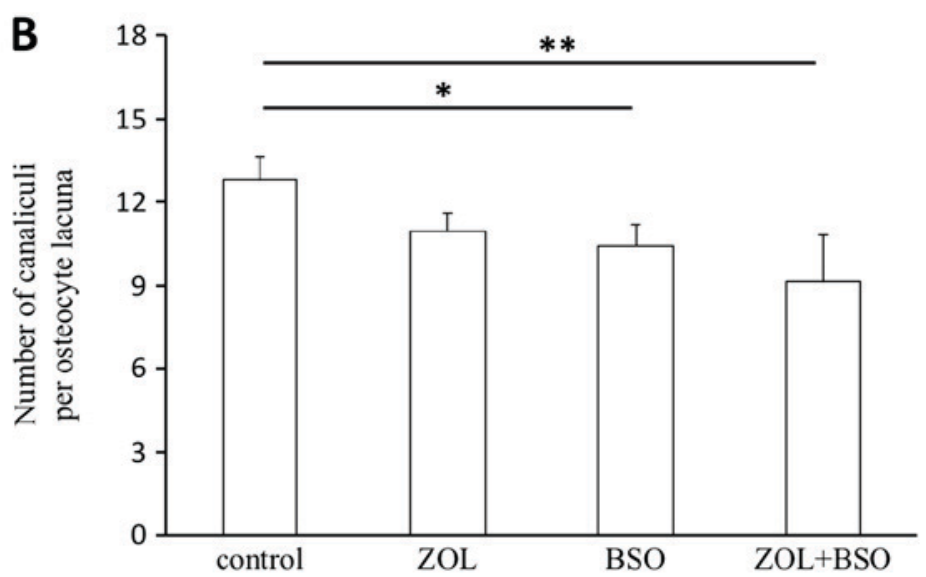

Figure 4. Canaliculi structure analysis. (A) AgNOR staining of osteocytic canaliculi in the palatal bone. Original magnification, $\mathrm{x} 400$. Scale bar, $50 \mu \mathrm{m}$. Insert presents a magnified photomicrograph of osteocyte lacuna. Scale bar, $10 \mu \mathrm{m}$. (B) The number of canaliculi per osteocyte lacuna in 10 randomly selected cells in their defined regions of interest. Magnification, $\mathrm{x} 400{ }^{*} \mathrm{P}<0.05$ and ${ }^{* *} \mathrm{P}<0.005$. ZOL, zoledronic acid; BSO, DL-buthionine-(S,R)-sulfoximine.

dendritic processes to communicate with each other, bone surface cells such as osteoblasts and osteoclasts, and vasculature cells (47). Osteocytes produce and secrete sclerostin and receptor activator of nuclear factor- $\kappa \mathrm{B}$ 
A

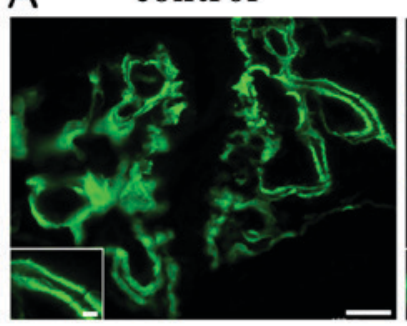

ZOL

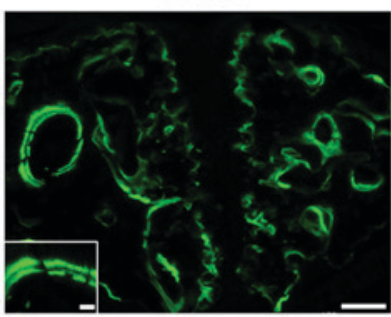

BSO

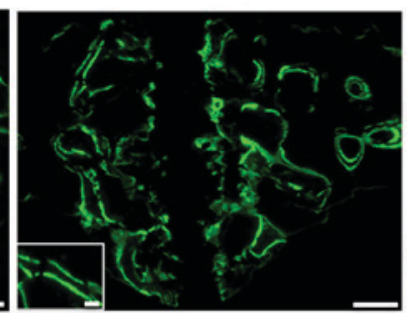

ZOL + BSO

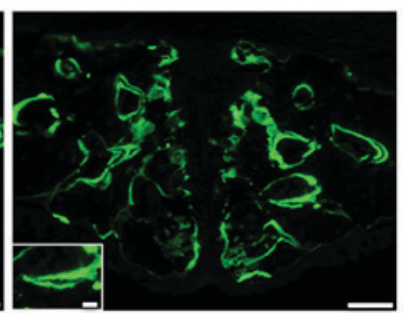

B

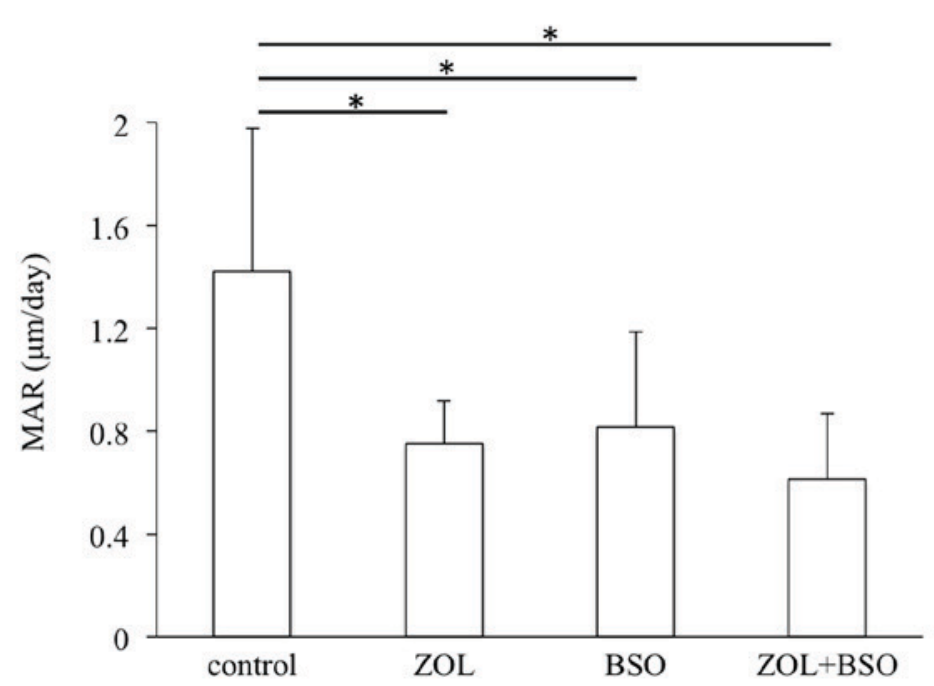

Figure 5. Fluorescence photomicrographs of calcein bone labeling. (A) Images of calcein double labeling of the palatal bone. Original magnification, x200. Scale bar, $50 \mu \mathrm{m}$. Insert presents a magnified photomicrograph of calcein double labels. Scale bar, $10 \mu \mathrm{m}$. (B) The MAR, defined as the distance between the midpoints of the double label divided by the number of days between calcein injections, was measured. * $\mathrm{P}<0.05$ vs. control. MAR, mineral apposition rate; ZOL, zoledronic acid; BSO, DL-buthionine-(S,R)-sulfoximine.

ligand to communicate indirectly with bone-associated cells. The osteocyte has a key role in regulating bone turnover. Busse et al (48) and Dunstan et al (49) previously revealed age-associated reduction in osteocyte viability. Kobayashi et al (50) recently demonstrated a similar reduction in both canalicular density and number in aged murine and oxidative-damaged osteocytes, supporting the hypothesis that aging and/or redox imbalances in osteocytes commonly exacerbate the impairment of osteocytic canalicular networks and reduce survival in mammals. The present study reported a $16 \%$ reduction in N.Ot.Ca/Ot.Lc. in the palatal bone with BSO treatment. Otherwise, the N.Ot. $\mathrm{Ca} / \mathrm{Ot}$.Lc. was slightly decreased by ZOL treatment, but not significantly. The MAR was also evaluated. The MAR was lower in the treatment groups than in the control group and was lowest in the ZOL+BSO group. These results suggest that $\mathrm{BP}$ and $\mathrm{BSO}$ treatments serve an important role in the inhibition of bone turnover following dentoalveolar surgery.

The presence of bacterial colonies around the surgical perforation was not evaluated. Howie et al (11) recently established a model for osteonecrosis of the jaw with zoledronate treatment following repeated major trauma, and there was no detectable bacterial colonization at 1 week following extraction in either the control or zoledronate-treated rats. The term 'osteonecrosis' in BRONJ is associated with aseptic necrosis. According to the 2014 position paper of the American Association of Oral and Maxillofacial Surgeons (42), stage 1 BRONJ does not comprise bacterial infection. Therefore, stage 1 BRONJ can be defined as an osteonecrosis type of BRONJ.

In conclusion, investigation of this model has demonstrated that osteonecrosis induced by BSO treatment was similar to that induced by ZOL treatment. ZOL treatment may primarily target inhibition of osteoclasts, and BSO treatment affects osteocytes. OS in osteocytes exacerbate the impairment of osteocytic canalicular networks. Both BPs and OS suppressed bone turnover. As a result, BPs and OS may induce osteonecrosis following invasive dentoalveolar surgery. OS has been demonstrated as an additional risk factor for the development of BRONJ.

\section{Acknowledgements}

The authors would like to thank Professor Takashi Daimon (Department of Medical Informatics, Hyogo College of Medicine, Nishinomiya, Japan) for assisting with the statistical analysis and Ms Shinobu Osawa for her technical assistance.

\section{Funding}

The present study was supported by JSPS KAKENHI [grant nos. 15K11332, 18K09825 (to KT), 18K17124 (JT), and 26861761 (MY)], and by a Grant-in-Aid for Researchers [Hyogo College of Medicine, 2016 (to KT)] and a Grant-in-Aid for Graduate Students [Hyogo College of Medicine, 2017 (to JT)]. 


\section{Availability of data and materials}

The datasets used and/or analyzed during the current study are available from the corresponding author on reasonable request.

\section{Authors' contributions}

JT, KT and HK conceived and designed the present study. JT, $\mathrm{KT}, \mathrm{HH}, \mathrm{MU}, \mathrm{HM}$ and MY performed the experiments. JT, $\mathrm{KT}, \mathrm{HH}, \mathrm{KY}, \mathrm{KN}$ and $\mathrm{HK}$ analyzed the data and performed statistical analysis. JT, KT, KN and HK wrote, reviewed and revised the manuscript. All authors read and approved the final manuscript.

\section{Ethics approval and consent to participate}

All animal experiments were conducted in compliance with the study protocol, which was reviewed by the Animal Care and Use Committee of Hyogo College of Medicine (Nishinomiya, Japan) in accordance with the Act on Welfare and Management of Animals (Law No. 105, Japan), the Standards Relating to the Care and Management of Laboratory Animals and Relief of Pain (Japanese Ministry of Environment, Notice No. 88, 2006), and the Fundamental Guidelines for Proper Conduct of Animal Experiment and Related Activities in Academic Research Institutions (Japanese Ministry of Education, Culture, Sports, Science and Technology, Notice No. 71, 2006). Our proposed study was approved by the committee under the institutional approval no. 16-078.

\section{Patient consent for publication}

Not applicable.

\section{Competing interests}

The authors declare that they have no competing interests.

\section{References}

1. Migliorati CA, Casiglia J, Epstein J, Jacobsen PL, Siegel MA and Woo SB: Managing the care of patients with bisphosphonate-associated osteonecrosis: An American Academy of Oral Medicine position paper. J Am Dent Assoc 136: 1658-1668, 2005.

2. Cheng A, Mavrokokki A, Carter G, Stein B, Fazzalari NL, Willson DF and Goss AN: The dental implications of bisphosphonates and bone disease. Aust Dent J 50 (4 Suppl 2): S4-S13, 2005.

3. Bertoldo F, Santini D and Lo Cascio V: Bisphosphonates and osteomylelitis of the jaw: A pathogenic puzzle. Nat Clin Pract Oncol 4: 711-721, 2007.

4. Licata AA: Discovery, clinical development, and therapeutic uses of bisphosphonates. Ann Pharmacother 39: 668-677, 2005.

5. Michaelson MD and Smith MR: Bisphosphonates for treatment and prevention of bone metastases. J Clin Oncol 23: 8219-8224, 2005.

6. Ruggiero SL, Dodson TB, Assael LA, Landesberg R, Marx RE and Mehrotra B; American Association of Oral and Maxillofacia Surgeons: American Association of Oral and Maxillofacial Surgeons position paper on bisphosphonate-related osteonecrosis of the jaws-2009 update. J Oral Maxillofac Surg 67 (Suppl 5): $2-12,2009$

7. Urade M, Tanaka N, Furusawa K, Shimada J, Shibata T, Kirita T, Yamamoto T, Ikebe T, Kitagawa Y and Fukuta J: Nationwide survey for bisphosphonate-related osteonecrosis of the jaws in Japan. J Oral Maxillofac Surg 69: e364-e371, 2011.
8. Doggrell SA: Clinical efficacy and safety of zoledronic acid in prostate and breast cancer. Expert Rev Anticancer Ther 9: 1211-1218, 2009.

9. Reid IR and Cornish J: Epidemiology and pathogenesis of osteonecrosis of the jaw. Nat Rev Rheumatol 8: 90-96, 2011.

10. Kim JH, Park YB, Li Z, Shim JS, Moon HS, Jung HS and Chung MK: Effect of alendronate on healing of extraction sockets and healing around implants. Oral Dis 17: 705-711, 2011.

11. Howie RN, Borke JL, Kurago Z, Daoudi A, Cray J, Zakhary IE, Brown TL, Raley JN, Tran LT, Messer R, et al: A model for osteonecrosis of the jaw with zoledronate treatment following repeated major trauma. PLoS One 17: e0132520, 2015.

12. Poubel VLDN, Silva CAB, Mezzomo LAM, De Luca Canto G and Rivero ERC: The risk of osteonecrosis on alveolar healing after tooth extraction and systemic administration of antiresorptive drugs in rodents: A systematic review. J Craniomaxillofac Surg 46: 245-256, 2018.

13. Advisory Task Force on Bisphosphonate-Related Ostenonecrosis of the Jaws, American Association of Oral and Maxillofacial Surgeons: American Association of Oral and Maxillofacial Surgeons position paper on bisphosphonate-related osteonecrosis of the jaws. J Oral Maxillofac Surg 65: 369-376, 2007.

14. Sonis ST, Watkins BA, Lyng GD, Lerman MA and Anderson KC: Bony changes in the jaws of rats treated with zoledronic acid and dexamethasone before dental extractions mimic bisphosphonate-related osteonecrosis in cancer patients. Oral Oncol 45: 164-172, 2009.

15. Hokugo A, Christensen R, Chung EM, Sung EC, Felsenfeld AL, Sayre JW, Garrett N, Adams JS and Nishimura I: Increased prevalence of bisphosphonate-related osteonecrosis of the jaw with vitamin D deficiency in rats. J Bone Miner Res 25: 1337-1349, 2010.

16. Takaoka K, Yamamura M, Nishioka T, Abe T, Tamaoka J, Segawa E, Shinohara M, Ueda H, Kishimoto $\mathrm{H}$ and Urade M: Establishment of an animal model of bisphosphonate-related osteonecrosis of the jaws in spontaneously diabetic torii rats. PLoS One 14: e0144355, 2015.

17. Bamias A, Kastritis E, Bamia C, Moulopoulos LA, Moulopoulos I, Bozasb G, Koutsoukou V, Gika D, Anaqnostopoulos A, Papadimitriou C, et al: Osteonecrosis of the jaw in cancer after treatment with bisphosphonates: Incidence and risk factors. J Clin Oncol 23: 8580-8587, 2005.

18. Marx RE, Sawatari Y, Fortin M and Broumand V: Bisphosphonate-induced exposed bone (osteonecrosis/osteopetrosis) of the jaws: Risk factors, recognition, prevention, and treatment. J Oral Maxillofac Surg 63: 1567-1575, 2005.

19. Jadu F, Lee L, Pharoah M, Reece D and Wang L: A retrospective study assessing the incidence, risk factors and comorbidities of pamidronate-related necrosis of the jaws in multiple myeloma patients. Ann Oncol 18: 2015-2019, 2007.

20. Almeida M, Han L, Martin-Millan M, Plotkin LI, Stewart SA, Roberson PK, Kousteni S, O'Brien CA, Bellido T, Parfitt AM, et al: Skeletal involution by age-associated oxidative stress and its acceleration by loss of sex steroids. J Biol Chem 282: 27285-27297, 2007.

21. Almeida M, Ambrogini E, Han L, Manolagas SC and Jilka RL: Increased lipid oxidation causes oxidative stress, increased peroxisome proliferator-activated receptor-gamma expression, and diminished pro-osteogenic Wnt signaling in the skeleton. J Biol Chem 284: 27438-27448, 2009.

22. Nojiri H, Saita Y, Morikawa D, Kobayashi K, Tsuda C, Miyazaki T, Saito M, Marumo K, Yonezawa I, Kaneko K, et al: Cytoplasmic superoxide causes bone fragility owing to low-turnover osteoporosis and impaired collagen cross-linking. J Bone Miner Res 26: 2682-2694, 2011.

23. Almeida M and O'Brien CA: Basic biology of skeletal aging: Role of stress response pathways. J Gerontol Biol Sci Med Sci 68: 1197-1208, 2013.

24. Domazetovic V, Marcucci G, Iantomasi T, Brandi ML and Vincenzini MT: Oxidative stress in bone remodeling: Role of antioxidants. Clin Cases Miner Bone Metab 14: 209-216, 2017.

25. Koçer G, Naziroğlu M, Çelik Ö, Önal L, Özçelik D, Koçer M and Sönmez TT: Basic fibroblast growth factor attenuates bisphosphonate-induced oxidative injury but decreases zinc and copper levels in oral epithelium of rat. Biol Trace Elem Res 153: 251-256, 2013.

26. Awodele O, Olayemi SO, Nwite JA and Adeyemo TA: Investigation of the levels of oxidative stress parameters in HIV and HIV-TB co-infected patients. J Infect Dev Ctries 6: 79-85, 2012. 
27. Lebreton F, van Schaik W, Sanguinetti M, Posteraro B, Torelli R, Lee Bras F, Vemeuil N, Zhang X, Giard JC, Dhalluin A, et al: AsrR is an oxidative stress sensing regulator modulating Enterococcus faecium opportunistic traits, antimicrobial resistance, and pathogenicity. PLoS Pathog 8: e1002834, 2012.

28. McDevitt CA, Ogunniyi AD, Valkov E, Lawrence MC, Kobe B, McEwan AG and Paton JC: A molecular mechanism for bacterial susceptibility to zinc. PLoS Pathog 7: e1002357, 2011

29. Moye-Rowley WS: Transcription factors regulating the response to oxidative stress in yeast. Antioxid Redox Signal 4: 123-140, 2002.

30. Khandelwal VK, Mitrofan LM, Hyttinen JM, Chaudhari KR, Buccione R, Kaarniranta K, Ravingerová T and Mönkkönen J: Oxidative stress plays an important role in zoledronic acid-induced autophagy. Physiol Res 63 (Suppl 4): S601-S612, 2014.

31. Kuribayashi M, Fujioka M, Takahashi KA, Arai Y, Ishida M, Goto T and Kubo T: Vitamin E prevents steroid-induced osteonecrosis in rabbits. Acta Orthop 81: 154-160, 2010

32. Ichiseki T, Matsumoto T, Nishino M, Kaneuji A and Katsuda S: Oxidative stress and vascular permeability in steroid-induced osteonecrosis model. J Orthop Sci 9: 509-515, 2004.

33. Ichiseki T, Kaneuji A, Katsuda S, Ueda Y, Sugimori T and Matsumoto T: DNA oxidation injury in bone early after steroid administration is involved in the pathogenesis of steroid-induced osteonecrosis. Rheumatology (Oxford) 44: 456-460, 2005.

34. Ichiseki T, Kaneuji A, Ueda Y, Nakagawa S, Mikami T, Fukui K and Matsumoto T: Osteonecrosis development in a novel rat model characterized by a single application of oxidative stress. Arthritis Rheum 63: 2138-2141, 2011.

35. Kobayashi Y, Hiraga T, Ueda A, Wang L, Matsumoto-Nakano M Hata K, Yatani H and Yoneda T: Zoledronic acid delays wound healing of the tooth extraction socket, inhibits oral epithelial cell migration, and promotes proliferation and adhesion to hydroxyapatite of oral bacteria, without causing osteonecrosis of the jaw, in mice. J Bone Miner Metab 28: 165-175, 2010.

36. Valavanidis A, Vlachogianni T and Fiotakis C: 8-hydroxy-2'deoxyguanosine (8-OHdG): A critical biomarker of oxidative stress and carcinogenesis. J Environ Sci Health C Environ Carcinog Ecotoxicol Rev 27: 120-139, 2009.

37. Jaiprakash A, Prasadam I, Feng JQ, Liu Y, Crawford R and Xiao Y: Phenotypic characterization of osteoarthritic osteocytes from the sclerotic zones: A possible pathological role in subchondral bone sclerosis. Int J Biol Sci 8: 406-417, 2012

38. Kawamoto T and Kawamoto K: Preparation of thin frozen sections from nonfixed and undecalcified hard tissues using Kawamoto's film method (2012). Methods Mol Biol 1130: $149-164,2014$
39. Dempster DW, Compston JE, Drezner MK, Glorieux FH, Kanis JA, Malluche H, Meunier PJ, Ott SM, Recker RR and Parfitt AM: Standardized nomenclature, symbols and units for bone histomorphometry: A 2012 update of the report of the ASBMR Histomorphometry Nomenclature Committee. J Bone Miner Res 28: 2-17, 2013.

40. Huja SS and Beck FM: Bone remodeling in maxilla, mandible, and femur of young dogs. Anat Rec (Hoboken) 291: 1-5, 2008.

41. Rodan GA and Martin TJ: Therapeutic approaches to bone diseases. Science 289: 1508-1514, 2000.

42. Hughes DE, Wright KR, Uy HL, Sasaki A, Yoneda T, Roodman GD, Mundy GR and Boyce BF: Bisphosphonates promote apoptosis in murine osteoclasts in vitro and in vivo. J Bone Miner Res 10: 1478-1487, 1995.

43. Selander KS, Mönkkönen J, Karhukorpi EK, Härkönen P, Hannuniemi R and Väänänen HK: Characteristics of clodronate-induced apoptosis in osteoclasts and macrophages. Mol Pharmacol 50: 1127-1138, 1996.

44. Hiroi-Furuya E, Kameda T, Hiura K, Mano H, Miyazawa K, Nakamaru Y, Watanabe-Mano M, Okuda N, Shimada J, Yamamoto $\mathrm{Y}$, et al: Etidronate (EHDP) inhibits osteoclastic-bone resorption, promotes apoptosis and disrupts actin rings in isolate-mature osteoclasts. Calcif Tissue Int 64: 219-223, 1999

45. Santini D, Vespasiani Gentilucci U, Vincenzi B, Picardi A, Vasaturo F, La Cesa A, Onori N, Scarpa S and Tonini G: The antineoplastic role of bisphosphonates: From basic research to clinical evidence. Ann Oncol 14: 1468-1476, 2003.

46. Ruggiero SL, Dodson TB, Fantasia J, Goodday R, Aghaloo T, Mehrotra B and O'Ryan F; American Association of Oral and Maxillofacial Surgeons: American Association of Oral and Maxillofacial Surgeons position paper on medication-related osteonecrosis of the jaw-2014 update. J Oral Maxillofac Surg 72: 1938-1956, 2014.

47. Bonewald LF: The amazing osteocyte. J Bone Miner Res 26: 229-238, 2011.

48. Busse B, Djonic D, Milovanovic P, Hahn M, Püschel K, Ritchie RO, Djuric M and Amling M: Decrease in the osteocyte lacunar density accompanied by hypermineralized lacunar occlusion reveals failure and delay of remodeling in aged human bone. Aging Cell 9: 1065-1075, 2010.

49. Dunstan CR, Somers NM and Evans RA: Osteocyte death and hip fracture. Calcif Tissue Int 53 (Suppl 1): S113-S117, 1993.

50. Kobayashi K, Nojiri H, Saita Y, Morikawa D, Ozawa Y, Watanabe K, Koike M, Asou Y, Shirasawa T, Yokote K, et al: Mitochondrial superoxide in osteocytes perturbs canalicular networks in the setting of age-related osteoporosis. Sci Rep 5: 9148,2015 